\title{
Latent Complete-Lattice Structure of Hilbert-Space Projectors
}

\author{
Fedor Herbut \\ Serbian Academy of Sciences and Arts, Belgrade, Serbia.E-mail: fedorh@sanu.ac.rs \\ Editors: Vinayak Jagadish \& Danko Georgiev \\ Article history: Submitted on January 19, 2019; Accepted on February 28, 2019; Published on March 1, 2019.
}

\section{(} o uncover the hidden complete-lattice structure of Hilbert-space projectors, which is not seen by the operator operations and relations (algebraically), resort is taken to the ranges of projectors (to subspaces-to geometry). Taking the range of a projector is completed into a bijection of all projectors onto all subspaces of any finite or countably infinite dimensional Hilbert space. As a second step, this basic bijection is upgraded into an isomorphism of partially ordered sets utilizing the sub-projector relation on the one hand, and the subspace relation on the other. As a third and final step, the basic bijection is further upgraded to isomorphism of complete lattices. The complete-lattice structure is derived for subspaces, then, using the basic bijection, it is transferred to the set of all projectors. Some consequences in the quantum-mechanical formalism are examined with particular attention to the infinite sums appearing in spectral decompositions of discrete self-adjoint operators with infinite spectra.

Quanta 2019; 8: 1-10.

(c) (1) This is an open access article distributed under the terms of the Creative Commons Attribution License CC-BY-3.0, which permits unrestricted use, distribution, and reproduction in any medium, provided the original author and source are credited.

\section{Introduction}

In this work, I provide a detailed review with proofs of a not well known part of the quantum-mechanical formalism that relates to the lattice structure of quantum logic $[1-4]$. The presentation consists of the following two parts.

Conceptual part. The formalism of quantum mechanics has been put on firm mathematical ground in the seminal book [5] of von Neumann. The deepest concern were the infinite sums in the spectral decompositions of some discrete self-adjoint operators. It will be shown in the present review that von Neumann's treatment is equivalently replaceable in the complete-lattice approach. The article starts by stating and proving some elementary properties of the quantum-mechanical formalism that will be needed in the sequel.

Technical part. The article is based on the so-called Dirac-von Neumann approach [5 6]. By 'Hilbert space' is meant in this article a complex finite or countably infinite dimensional Hilbert space. The Dirac notation [7] is used throughout. By basis in any subspace of a Hilbert space, or in the latter itself, is meant any ortho-normal set (set of orthogonal and normalized vectors, i.e., of vectors of norm 1) that spans the subspace (all elements of the subspace can be expanded in the basis) [8]. The left-hand side and the right-hand side of an equality (...) will sometimes be denoted by LHS(...) and RHS(...) respectively. Occasionally, to the right of a number the symbol ' $x$ ' will be put for emphasis. 


\section{The basic bijection}

The ranges of (linear) operators are, in general, so-called linear manifolds (sets closed under all finite linear combinations). They are in general not closed under the limit operation. (There may exist a convergent infinite sequence in the linear manifold the limit of which does not belong to the manifold). If $\hat{O}$ and $\mathcal{R}(\hat{O})$ are a linear operator and its range, then one takes the so-called topological closure $\overline{\mathcal{R}}(\hat{O})$, (the least-dimensional subspace of which $\mathcal{R}(\hat{O})$ is a subset), which enlarges the range with all the limiting elements. For projectors this is not necessary.

To begin with, one should be reminded that the scalar product in Hilbert space $\mathcal{H}$ has the property of so-called positive semi-definiteness:

$$
\begin{gathered}
\forall|\psi\rangle \in \mathcal{H}: \quad\langle\psi \| \psi\rangle \geq 0, \\
\langle\psi \| \psi\rangle=0 \quad \Leftrightarrow \quad|\psi\rangle=0,
\end{gathered}
$$

where ' $\Leftrightarrow$ ' denotes logical implication in both directions. Property (1) makes possible the definition of the norm of every vector

$$
|\psi\rangle: \quad \| \psi\rangle \| \equiv(\langle\psi \| \psi\rangle)^{1 / 2} .
$$

Lemma 1. If $|\psi\rangle$ and $\hat{P}$ are an arbitrary vector and an arbitrary projector, then

A)

$$
\langle\psi|\hat{P}| \psi\rangle \geq 0,
$$

and the equality is satisfied if and only if $\hat{P}|\psi\rangle=0$.

$B)$ The norm of the projection satisfies $\| \hat{P}|\psi\rangle\|\leq\||\psi\rangle\rangle \|$, and the equality is valid if and only if $\hat{P}|\psi\rangle=|\psi\rangle$.

Proof. A) The claim follows from the fact that projectors are idempotent $\left(\hat{P}^{2}=\hat{P}\right)$ and self-adjoint $\left(\hat{P}=\hat{P}^{\dagger}\right.$, where the dagger denotes adjoining). $\operatorname{LHS}(2)=(\langle\psi| \hat{P})(\hat{P}|\psi\rangle)$. Semi-definiteness of the scalar product then implies the claim. B) Utilizing $\hat{P}^{\perp} \equiv \hat{I}-\hat{P}$ ( $\hat{I}$ being the identity operator), the ortho-complementary projector (physically the opposite event or property), one has

$$
\||\psi\rangle\left\|^{2}=\left\langle\psi\left|\left(\hat{P}+\hat{P}^{\perp}\right)\right| \psi\right\rangle \geq\langle\psi|\hat{P}| \psi\rangle=\right\| \hat{P}|\psi\rangle \|^{2} .
$$

The inequality follows of course from Lemma 14A. One can see from the first equality in the proof that one can have equality in the claimed inequality in the lemma if and only if $\left\langle\psi\left|\hat{P}^{\perp}\right| \psi\right\rangle=0$. Then also $\| \hat{P}^{\perp}|\psi\rangle \|=0$ implying (cf (1)) $\hat{P}^{\perp}|\psi\rangle=0$ equivalent to $\hat{P}|\psi\rangle=|\psi\rangle$.

Lemma 2. Projectors are continuous: Let $\hat{P}$ be a projector and let $\{|m\rangle: m=1,2, \ldots \infty\}$ be a convergent infinite sequence converging to $|\psi\rangle:|\psi\rangle=\lim _{m \rightarrow \infty}|m\rangle$. Then $\hat{P} \lim _{m \rightarrow \infty}|m\rangle=\lim _{m \rightarrow \infty} \hat{P}|m\rangle$.
Proof. Follows from Lemma $1 \mathrm{~B}$. We have

$$
\| \hat{P}|m\rangle-\hat{P}|\psi\rangle\|=\| \hat{P}(|m\rangle-|\psi\rangle)\|\leq|\| m\rangle-|\psi\rangle\| \leq \epsilon
$$

if $m$ is large enough. Here $\epsilon$ is an arbitrarily small positive number (defining convergence), and $\||m\rangle-|\psi\rangle||$ is the distance between the two vectors.

Now we are prepared to start establishing the claimed bijection between projectors and subspaces. We begin by showing that the projector ranges are subspaces. One should be reminded that by the 'range' $\mathcal{R}(\hat{P})$ of a projector $\hat{P}$ is meant the set $\{\hat{P}|\psi\rangle:|\psi\rangle \in \mathcal{H}\}$ of all vectors that can be written in the form $\hat{P}|\phi\rangle$. The ortho-complement (set of all vectors that are orthogonal to all vectors in the range) of the range $\mathcal{R}(\hat{P})$ is called the null space of the projector $\hat{P}$.

Proposition 1. If $\hat{P}$ is a projector and $\{|m\rangle: m=1,2, \ldots \infty\}$ is a convergent infinite sequence in the range $\mathcal{R}(\hat{P})$ converging to $|\psi\rangle$, then $|\psi\rangle$ necessarily belongs to the range $\mathcal{R}(\hat{P})$.

Proof. Follows from continuity of the projector. Applying $\hat{P}$ to $|\psi\rangle=\lim _{m \rightarrow \infty}|m\rangle$, one obtains

$$
\hat{P}|\psi\rangle=\hat{P} \lim _{m \rightarrow \infty}|m\rangle=\lim _{m \rightarrow \infty} \hat{P}|m\rangle=\lim _{m \rightarrow \infty}|m\rangle .
$$

Since the limit is unique, one has $\hat{P}|\psi\rangle=|\psi\rangle$. This is equivalent to the claimed relation $|\psi\rangle \in \mathcal{R}(\hat{P})$ because the range is the set of all vectors that can be written in the form $\hat{P}|\phi\rangle$.

Thus, the range $\mathcal{R}(\hat{P})$ of any projector $\hat{P}$ is a subspace, and it is precisely the subspace onto which $\hat{P}$ projects the entire space.

If $\hat{P}$ is a projector, its null space is the orthocomplement of the range, or equivalently, the range of the ortho-complementary projector $\hat{P}^{\perp}(\equiv \hat{I}-\hat{P})$.

Proposition 2. If $\hat{P}$ is a projector, then its range is its eigen-subspace corresponding to the eigenvalue 1. The null space of $\hat{P}$ is its 0-eigen-subspace.

Proof. Follows from idempotency. If $|\psi\rangle \in \mathcal{R}(\hat{P})$, then there exists $|\phi\rangle$ such that $|\psi\rangle=\hat{P}|\phi\rangle$. Applying $\hat{P}$ to this equality, one obtains

$$
\hat{P}|\psi\rangle=\hat{P}^{2}|\phi\rangle=\hat{P}|\phi\rangle=|\psi\rangle=1 \times|\psi\rangle .
$$

As to the null space, if $|\psi\rangle \in \mathcal{R}(\hat{I}-\hat{P})$ is valid, then $(\hat{I}-\hat{P})|\psi\rangle=|\psi\rangle$, implying $-\hat{P}|\psi\rangle=0$.

Corollary 1. No two distinct projectors can have the same range. 
Proof. Follows immediately from Proposition 2 . Same range would mean same 1-eigen-subspace (in which they would both act as the identity operator). Same range would imply same null space, i.e., same 0 -eigen-subspace (in which they would both act as the zero operator). Then the two projectors could not be distinct.

We see from Proposition 1 and Corollary 1 that associating with a projector its range is an injection, i.e., a bijection of the set of all projectors into the set of all subspaces. Equivalently put, we have a bijection of the former set onto a proper or improper subset of the latter set. Hence, what remains to be done is to show that there is no subspace that is not the range of a projector.

Proposition 3. If $S$ is an arbitrary subspace, then there exists a projector the range of which it is. We choose an arbitrary basis in $S:\{|m\rangle: m=1,2, \ldots, M\}, M$ being an integer or $\infty$ (when $S$ is finitely or infinitely dimensional respectively). Defining $\hat{P} \equiv \sum_{m=1}^{M}|m\rangle\langle m|$, we obtain a unique projector. The range of $\hat{P}$ is $S: \mathcal{R}(\hat{P})=S$.

Proof. Let $|\psi\rangle$ be an arbitrary vector in $S$. Then one can expand it in the chosen basis:

$$
|\psi\rangle=\sum_{m=1}^{M}\langle m \| \psi\rangle|m\rangle=\sum_{m=1}^{M}|m\rangle\langle m \| \psi\rangle=\hat{P}|\psi\rangle .
$$

Thus, $|\psi\rangle \in \mathcal{R}(\hat{P})$. Since $|\psi\rangle$ is an arbitrary element of $S$, We have obtained the first half of our proof: $\mathrm{S} \subseteq \mathcal{R}(\hat{P})$.

Next, we take an arbitrary vector $|\phi\rangle$. Then $\hat{P}|\phi\rangle$ is an arbitrary element of $\mathcal{R}(\hat{P})$. We have

$$
\hat{P}|\phi\rangle=\sum_{m=1}^{M}|m\rangle\langle m \| \phi\rangle=\sum_{m=1}^{M}(\langle m \| \phi\rangle) \times|m\rangle .
$$

Hence, $\hat{P}|\phi\rangle \in S$. This provides the other half of the proof: $\mathcal{R}(\hat{P}) \subseteq S$. If two sets are subsets of each other, then they are equal. Since no two different projectors can have the same range (cf Corollary 1), the projector $\hat{P}$ is unique though the basis in the subspace $S$ can be chosen in (infinitely) many ways.

We have thus completed the proof of the following theorem. Taking the range of a projector will be called the basic bijection in it. The set of all projectors will be denoted by $\mathcal{S}_{P}$ and the set of all subspaces of the given Hilbert space by $\mathcal{S}_{S}$. We shall refer to projector and its range, or vice versa, as corresponding entities.

Theorem 1. Taking the range of a projector is a bijection (one-to-one map) of the set of all projectors $\mathcal{S}_{P}$ onto the set of all subspaces $\mathcal{S}_{S}$. The inverse map of the basic bijection can be obtained by specifying any basis $\{|m\rangle, m=1,2, \ldots M\}$ in the chosen subspace, then
$\hat{P} \equiv \sum_{m}|m\rangle\langle m|$ is the projector the range of which the given subspace is.

This establishes the claimed one-to-one map between the set of all subspaces $\mathcal{S}_{S}$ and the set of all projectors $\mathcal{S}_{P}$. Since we use projectors more often than subspaces, we can write the latter simply as ranges of the former.

\section{Upgrading the basic bijection to an isomorphism between two partially ordered sets}

Definition 1. A binary relation in a set $\mathcal{A}$ is called a partial order, and often denoted by $a \leq b, a, b \in \mathcal{A}$, if the relation has the properties of reflexivity: $a \leq a, \forall a \in \mathcal{A}$; symmetry: if $a \leq b$ and $b \leq a, a, b \in \mathcal{A}$, are both valid, then $b=a$; and finally transitivity: if $a \leq b$ and $b \leq c, a, b, c \in \mathcal{A}$, then $a \leq c$ is implied. A set $\mathcal{A}$ in which a partial order is defined is called a partiallyordered set (poset). (The interested reader may learn more about the partial-order relation in [2,9, 10].)

Let $\hat{P}$ and $\hat{Q}$ be two projectors, and let $\hat{P} \leq \hat{Q}$, which is symbolic for $\hat{P} \hat{Q}=\hat{P}$ or equivalently $\hat{Q} \hat{P}=\hat{P}$ (as seen by adjoining), the so-called sub-projector relation, be valid. In case of a sub-projector relation, we shall use the practical terminology: $\hat{P}$ ejects or absorbs $\hat{Q}$.

The physical meaning of $\hat{P} \leq \hat{Q}$, if the projectors mean events, is the fact that if $\hat{P}$ occurs, so does $\hat{Q}$.

Lemma 3. The set $\mathcal{S}_{P}$ of all projectors in a Hilbert space is a partially ordered set in which the partial-order relation is the sub-projector relation.

Proof. Reflexivity: $\hat{P}=\hat{P} \hat{P}$ (idempotency viewed as ejection). Symmetry: If $\hat{P} \hat{Q}=\hat{P}$ and $\hat{Q} \hat{P}=\hat{Q}$ are valid, then (using ejection and absorbtion)

$$
\hat{Q}=\hat{Q} \hat{P}=\hat{P} .
$$

Transitivity: If $\hat{P}, \hat{Q}$, and $\hat{R}$ are projectors such that $\hat{P}=\hat{P} \hat{Q}$ and $\hat{Q}=\hat{Q} \hat{R}$, then (using ejection twice and absorbtion once, resulting in ejection):

$$
\hat{P}=\hat{P} \hat{Q}=\hat{P} \hat{Q} \hat{R}=\hat{P} \hat{R}
$$

is valid.

Lemma 4. The set of all subspaces $\mathcal{S}_{S}$ of a Hilbert space is a partially ordered set in which the partial-order relation is the subset relation ' $\subseteq$ '.

Proof. It is straightforward. 
Theorem 2. (Isomorphism of partially-ordered sets) Both the basic bijection (cf Theorem 1) and its inverse map preserve the partial-order relation. This fact makes the set of all projectors $\mathcal{S}_{P}$ and that of all subspaces $\mathcal{S}_{S}$ (in any Hilbert space) isomorphic partially ordered sets.

Proof. Let $\hat{P}$ and $\hat{Q}$ be projectors and let $\hat{P} \leq \hat{Q}$. Further, let $|\psi\rangle$ be an arbitrary vector. Then $\hat{P}|\psi\rangle$ is an arbitrary element of the range $\mathcal{R}(\hat{P})$. Also $\hat{P}|\psi\rangle=\hat{Q} \hat{P}|\psi\rangle \in \mathcal{R}(\hat{Q})$ is valid. This proves preservation of the partial-order relation in the direction from projectors to the corresponding subspaces. Next, let $\hat{P}$ and $\hat{Q}$ be such projectors that $\mathcal{R}(\hat{P}) \subseteq \mathcal{R}(\hat{Q})$, and let $|\psi\rangle$ be an arbitrary vector. Then $\hat{P}|\psi\rangle \in \mathcal{R}(\hat{P})$, and, on account of the assumption, then also $\hat{P}|\psi\rangle \in \mathcal{R}(\hat{Q})$ is valid. This implies $\hat{Q} \hat{P}|\psi\rangle=\hat{P}|\psi\rangle$ (cf Proposition 2). Since $|\psi\rangle$ is an arbitrary vector, this finally gives $\hat{Q} \hat{P}=\hat{P}$. Thus, the partial-order relation is preserved also in the direction from subspaces to corresponding projectors.

What follows is an obvious consequence of the isomorphism in Theorem 2 .

Corollary 2. Whatever is valid in one of the isomorphic sets $\left(\mathcal{S}_{S}\right.$ or $\left.\mathcal{S}_{P}\right)$ in terms of the isomorphic partial-order relations, is necessarily valid also in the other of the isomorphic sets in terms of the isomorphic entities.

This kind of mirror-image property of the isomorphism will play a crucial role in the present investigation.

\section{Towards further upgrading the basic bijection}

Definition 2. An element $u$ of a partially ordered set $\mathcal{A}$ is called an upper bound of a given subset $\left\{b_{m}: m \in \mathcal{M}\right\}$ of one, two, or any finite or infinite number of elements (in $\mathcal{M}$ ) if $\forall m \in \mathcal{M}: b_{m} \leq u$ is valid. An upper bound of a set is called its least upper bound (or supremum), denoted by lub (or sup) if every upper bound of the set is an upper bound also of the lub. The lub is the minimal upper bound [11]. Symmetrically, an element $l$ is called a lower bound of the given set if $\forall m \in \mathcal{M}: l \leq b_{m}$ is satisfied. It is a greatest lower bound (or infimum) denoted by glb (or inf) if every lower bound of the set is a lower bound also of the glb (maximal lower bound) [9].

Definition 3. A partially ordered set $\mathcal{A}$ is a lattice if every two elements $a, b$ in it have both a common lub and a common glb [2,12].

Remark 1 . In any partially-ordered set the lub and the glb, if they exist, they are unique. This is seen if one assumes that, e.g., $c$ and $c^{\prime}$ are least upper bounds for $a, b$, then both $c \leq c^{\prime}$ and $c^{\prime} \leq c$ would be valid implying $c=c^{\prime}$ due to the symmetry requirement in the definition of a partial-order relation (cf Definition 11). Analogously one proves that also the glb has to be unique.

Lemma 5. If $\left\{a_{1}, a_{2}, \ldots, a_{N}\right\}$ is any finite subset of a lattice $\mathcal{A}$, then it has both a lub and a glb.

Proof. Uses mathematical induction. Let us assume that we already know that all subsets of $N-1$ elements do have a lub. Let that of the subset $\left\{a_{1}, a_{2}, \ldots, a_{(N-1)}\right\}$ of the given subset $\left\{a_{1}, a_{2}, \ldots, a_{N}\right\}$ be $\bar{a}_{(N-1)}$. Further, let the common lub of $\bar{a}_{(N-1)}$ and $a_{N}$ be $\bar{a}_{N}$. Then we prove that it is also the lub of $\left\{a_{1}, a_{2}, \ldots, a_{N}\right\}$.

Owing to the transitivity property of the partial-order relation, the element $\bar{a}_{N}$ is obviously an upper bound of the given subset $\left\{a_{1}, a_{2}, \ldots, a_{N}\right\}$.

Let $u$ be another upper bound of the given subset $\left\{a_{1}, a_{2}, \ldots, a_{N}\right\}$. Then it is an upper bound also of $\left\{a_{1}, a_{2}, \ldots, a_{(N-1)}\right\}$. Since $\bar{a}_{(N-1)}$ is the lub of the latter set, $u$ is an upper bound also of $\bar{a}_{N-1}$. Thus, $u$ is an upper bound of both $\bar{a}_{(N-1)}$ and $a_{N}$. Since $\bar{a}_{N}$ is the lub of these two, $u$ is an upper bound of $\bar{a}_{N}$.

This argument makes $\bar{a}_{N}$ the lub of the given subset $\left\{a_{1}, a_{2}, \ldots, a_{N}\right\}$.

Mathematical induction says that if a claim is valid for the first member of an infinite sequence, and if from the validity of the claim for the $(N-1)$ th member $(N \geq 2)$ follows the validity for the $N$ th member, then the claim is valid for the entire sequence. Our proof is in this way completed.

Remark 2. The lattice property cannot guarantee the existence of a lub and a glb for infinite subsets. This must be an explicit requirement in the definition.

Definition 4. A partially-ordered set $\mathcal{A}$ is a complete lattice if every subset, being finite, or countably infinite or uncountably infinite, has a lub and a glb [2,13].

Lemma 6. Let $\mathcal{B}$ be an arbitrary subset of a partiallyordered set $\mathcal{A}$ that is a complete lattice. Let further $\mathcal{U}$ and $\mathcal{L}$ be the set of all upper bounds and that of all lower bounds of the given subset $\mathcal{B}$. Then the equalities $\operatorname{lu} b(\mathcal{L})=\operatorname{glb}(\mathcal{B})$ and $\operatorname{lu} b(\mathcal{B})=g l b(\mathcal{U})$ are valid.

Proof. Let $a$ be the glb of $\mathcal{B}$. Since it is a lower bound, it belongs to $\mathcal{L}$. Since it is the greatest, it is the maximal element of $\mathcal{L}$. This very fact makes it the least upper bound of $\mathcal{L}$. This proves the first relation in the Lemma. Analogously one proves also the second relation in the Lemma. 


\subsection{Further upgrading the basic bijection to isomorphism of complete lattices}

The entire subsection hinges on the following claim. On account of the simple but powerful properties of $\mathcal{S}_{S}$, the set of all subspaces of a given Hilbert space, the claim reveals that $\mathcal{S}_{S}$ is, what is called, a lower half complete lattice (in which by definition every subset has a glb but not necessarily also a lub in it).

Proposition 4. In $\mathcal{S}_{S}$ the partial-order relation is the inclusion relation ' $\subseteq$ ' between sets. Let $\mathcal{A}=\left\{S_{m}: \forall m \in \mathcal{M}\right\}$ be any set of subspaces. Then the intersection $\mathcal{B}=\bigcap_{m \in \mathcal{M}} S_{m}$ is the glb of $\mathcal{A}\left(\right.$ in $\left.\mathcal{S}_{S}\right)$.

Proof. We first establish that $\mathcal{B}$ is a subspace. Let $\left\{a_{1}, a_{2}, \ldots, a_{K}\right\}$ be any finite set of elements in $\mathcal{B}$, and let $\left\{\alpha_{1}, \alpha_{2}, \ldots, \alpha_{K}\right\}$ an arbitrary set of corresponding complex numbers. Then $\sum_{k=1}^{K} \alpha_{k} a_{k}$ is an arbitrary linear combination of elements in $\mathcal{B}$.

Owing to the definition of $\mathcal{B}$, all the elements $\left\{a_{1}, a_{2}, \ldots, a_{K}\right\}$ belong to each subspace in $\mathcal{A}$. Then so does each linear combination $\sum_{k=1}^{K} \alpha_{k} a_{k}$. Hence the same is valid for the intersection $\mathcal{B}$. Next, let $\left\{a_{n}: n=1,2, \ldots, \infty\right\}$ be a sequence in $\mathcal{B}$ converging to $b$. As above, the sequence belongs to each subspace $S_{m}$, which, being closed under taking the limit, contains also the limit element $b$. Then again, due to the definition of $\mathcal{B}$, it follows that $b$ belongs also to $\mathcal{B}$. This makes $\mathcal{B}$ closed under taking the limit. Therefore, $\mathcal{B}$ is a subspace.

Further, we point out the obvious fact that the intersection $\mathcal{B}$ is a lower bound of $\mathcal{A}$.

Finally, we take an arbitrary lower bound $S$ of $\mathcal{A}$. Let the vector $s$ be an arbitrary element of $S$. Since now the partial-order relation is the inclusion relation between sets, $s$ belongs to each of the subspaces in $\mathcal{A}$, hence also to $\mathcal{B}$. This makes $S$ a lower bound of $\mathcal{B}$, and $\mathcal{B}$ the glb of $\mathcal{A}$ as claimed.

The next claim shows that the properties of $\mathcal{S}_{S}$ are so powerful that the (just proved) lower half complete-lattice structure of $\mathcal{S}_{S}$ determines its upper half complete-lattice structure, i.e., makes $\mathcal{S}_{S}$ a complete lattice.

Proposition 5. Let $\mathcal{A}$ be an arbitrary set of subspaces (in any given Hilbert space). It has both a glb and a lub. Hence $\mathcal{S}_{S}$, the set of all subspaces, is a complete lattice.

Proof. Let $\mathcal{U}$ be the set of all upper bounds of $\mathcal{A}$ in $\mathcal{S}_{S}$, i.e., the set of all subspaces such that each subspace belonging to $\mathcal{A}$ is a subspace of each subspace belonging to $\mathcal{U}$. Having in mind Lemma 6, in particular $\operatorname{glb}(\mathcal{U})=\operatorname{lub}(\mathcal{A})$, which is valid in any complete lattice, we take the intersection $\bigcap_{S \in \mathcal{U}} S$, where $S$ denotes a subspace. We know from Proposition 4 that this intersection is the glb of $\mathcal{U}$. Hence we define

$$
\operatorname{lub}(\mathcal{A}) \equiv \bigcap_{S \in \mathcal{U}} S .
$$

The definition is correct only if the RHS(3) is (i) a subspace, (ii) an upper bound of $\mathcal{A}$, and (iii) the minimal one among the upper bounds. Item (i) was proved in the proof of Proposition 4. To prove item (ii), we resort to the concrete properties of subspaces. Let $a$ be an arbitrary element of (vector in) an arbitrary subspace belonging to (being element of) $\mathcal{A}$, and let $S$ be an arbitrary subspace that is an upper bound of $\mathcal{A}$. It follows that $a \in S$. Since $S$ is an arbitrary element of $\mathcal{U}$, it further follows that $a \in \operatorname{RHS}(3)$. This makes RHS(3) an upper bound of $\mathcal{A}$, i.e., an element of $\mathcal{U}$. (iii) That the intersection is the minimal element of $\mathcal{U}$ is obvious.

There is an important consequence of the preceding proposition.

Theorem 3. In the given Hilbert space the set of all projectors $\mathcal{S}_{P}$ is a complete lattice in terms of its partialorder relation, the sub-projector relation. This upgrades the basic bijection (cf Theorem (1) into an isomorphism of complete lattices.

Proof. Since, according to Proposition 5 , in the given Hilbert space the set of all subspaces $\mathcal{S}_{S}$ is a complete lattice in terms of its partial-order relation (inclusion), and since the basic bijection (cf Theorem 1 preserves the partial-order relation ( $\mathrm{cf}$ Theorem 2), also the set of all projectors $\mathcal{S}_{P}$ is a complete lattice in terms of its partial-order relation, the sub-projector relation.

One should note that Theorem 3 gives confirmation and explicit expression to the title of this article.

Now we make a small deviation to a pragmatic property of complete lattices.

Corollary 3. If $\mathcal{A}=\bigcup_{m \in \mathcal{M}} \mathcal{B}_{m}$ is an arbitrary union of arbitrary sets $\mathcal{B}_{m}$ of projectors, then

$$
\operatorname{lub}(\mathcal{A})=\operatorname{lub}\left\{\operatorname{lub}\left(\mathcal{B}_{m}\right): m \in \mathcal{M}\right\},
$$

and symmetrically

$$
\left.g l b(\mathcal{A})=\operatorname{glb}\left\{g l b\left(\mathcal{B}_{m}\right): m \in \mathcal{M}\right)\right\} .
$$

Proof. First we show that the left-hand side of (4) is an upper bound of the right-hand side, i.e. RHS(4) $\leq$ LHS(4). (Here ' $\leq$ ' denotes the partial-order relation in $\mathcal{S}_{P}$, i.e., the sub-projector relation.) Since $\forall m \in \mathcal{M}: \mathcal{B}_{m} \subseteq \mathcal{A}$, and LHS(4) is an upper bound of $\mathcal{A}$ (of each projector in $\mathcal{A}$ ), $\operatorname{LHS}(4)$ is an upper bound also for each $\mathcal{B}$. This makes it an upper bound of each $\operatorname{lub}(\mathcal{B})$ and further of RHS(4). 
Next we prove that the RHS(4) is an upper bound of the LHS(4): LHS(4) $\leq$ RHS(4). Since the RHS(4) is an upper bound of each $\operatorname{lu} b\left(\mathcal{B}_{m}\right)$, it is an upper bound of each $\mathcal{B}_{m}$ (due to transitivity), then also of their union $\mathcal{A}$, and finally of LHS(4). The two opposite relations RHS(4) $\leq$ LHS(4) and LHS(4) $\leq$ RHS(4), on account of the symmetry requirement in the definition of partial order (cf Definition 11), imply the claimed equality LHS(4) $=$ RHS(4). The symmetric relation (5) is proved analogously (symmetrically).

One should note that if on the RHS(4) one of the $\operatorname{lu} b\left(\mathcal{B}_{m}\right)$ is the identity operator $\hat{I}$, then $\operatorname{lu} b \mathcal{A}=\hat{I}$. Symmetrically, if on the RHS(5) one of the $g l b\left(\mathcal{B}_{m}\right)$ is the zero operator $\hat{0}$, then $g l b \mathcal{A}=\hat{0}$. (The interested reader will hopefully turn to much more use the pragmatic completelattice properties in Corollary 3 , )

\section{Preservation of orthogonality and more}

Let the reader be reminded that orthogonality of two subspaces $S$ and $S^{\prime}$, i.e., when each vector in $S$ is orthogonal to each vector in $S^{\prime}$, is denoted by the perpendicularity symbol ' $\perp$ ': $S \perp S^{\prime}$.

Proposition 6. The basic bijection ( $c f$ Theorem 11) preserves orthogonality in both directions.

Proof. Let $\hat{P}$ and $\hat{Q}$ be orthogonal projectors $\hat{P} \hat{Q}=\hat{0}$. Let further $|\psi\rangle$ and $|\phi\rangle$ be arbitrary vectors, implying that $\hat{P}|\psi\rangle$ and $\hat{Q}|\phi\rangle$ are arbitrary elements of the ranges $\mathcal{R}(\hat{P})$ and $\mathcal{R}(\hat{Q})$ respectively. Using the mathematical notation for the scalar product this time (together with Dirac notation) because it is more transparent, one has $(\hat{P}|\psi\rangle, \hat{Q}|\phi\rangle)=$ $(|\psi\rangle, \hat{P} \hat{Q}|\phi\rangle)=0$. Therefore each vector from $\mathcal{R}(\hat{P})$ is orthogonal to each element of $\mathcal{R}(\hat{Q})$ proving that the basic bijection preserves orthogonality in the direction from projector to subspace.

Utilizing the same entities in the same notation as in the preceding passage, we start with $(\hat{P}|\psi\rangle, \hat{Q}|\phi\rangle)=0$ (expressing orthogonality of the ranges). But, on account oh the arbitrariness of the vectors $|\psi\rangle$ and $|\phi\rangle$, $0=(|\psi\rangle, \hat{P} \hat{Q}|\phi\rangle)$ implies $\hat{P} \hat{Q}=\hat{0}$. Thus, orthogonality is preserved in both directions as claimed.

\subsection{Orthogonal sums and their preservation}

Remark 3. One says that one has a set of orthogonal projectors if every two of them are orthogonal.
Lemma 7. If $N$ is a natural number $N \geq 2$, and if $\left\{\hat{P}_{n}: n=1,2, \ldots, N\right\}$ is a finite set of orthogonal projectors, then

$$
\hat{P}^{N} \equiv \sum_{n=1}^{N} \hat{P}_{n}=\operatorname{lub}\left\{\hat{P}_{n}: n=1,2, \ldots, N\right\} .
$$

Proof. First we establish that $\hat{P}^{N}=\sum_{n=1}^{N} \hat{P}_{n}$ is an upper bound of $\left\{\hat{P}_{n}: n=1,2, \ldots, N\right\}$. This means that we prove $\hat{P}_{n} \leq \hat{P}^{N}, n=1,2, \ldots, N$, which is symbolic for $\left(\sum_{n^{\prime}=1}^{N} \hat{P}_{n^{\prime}}\right) \hat{P}_{n}=\hat{P}_{n}, n=1,2, \ldots, N$. The validity of this claim follows immediately from the orthogonality and idempotency of projectors.

Next we establish that $\hat{P}^{N}$ is the minimal upper bound for the given set of projectors. Let a projector $\hat{Q}$ also be an upper bound of $\left\{\hat{P}_{n}: n=1,2, \ldots, N\right\}$. Then $\hat{Q} \hat{P}_{n}=\hat{P}_{n}, n=1,2, \ldots, N$. But then obviously also $\hat{Q} \sum_{n=1}^{N} \hat{P}_{n}=\sum_{n=1}^{N} \hat{P}_{n}$ is satisfied.

One often says 'an orthogonal set of projectors' (or subspaces) instead of 'a set of orthogonal projectors' (or subspaces).

Remark 4. It is easily seen that if $\left\{\hat{P}_{n}: n=1,2, \ldots, \infty\right\}$ is a countably infinite set of orthogonal projectors, then the corresponding sequence of sums $\left\{\sum_{n=1}^{N} \hat{P}_{n}: N=1,2, \ldots, \infty\right\}$ is a sequence of ascending projectors:

$$
\sum_{n=1}^{N} \hat{P}_{n} \leq \sum_{n=1}^{N^{\prime}} \hat{P}_{n} \quad \text { if } N \leq N^{\prime} .
$$

Von Neumann has shown in his book [5] on foundations of quantum mechanics that every ascending sequence of projectors has a projector limit. This projector is then, according to von Neumann [5] (and also according to the standard quantum-mechanical formalism) by definition the (countably) infinite orthogonal sum of the given projectors

$$
\hat{P}_{n} \hat{P}_{n^{\prime}}=\hat{0} \text { if } n \neq n^{\prime} \Rightarrow \sum_{n=1}^{\infty} \hat{P}_{n} \equiv \lim _{N \rightarrow \infty} \sum_{n=1}^{N} \hat{P}_{n} .
$$

Theorem 4. Let $\left\{\hat{P}_{n}: n=1,2, \ldots, \infty\right\}$ be a countably infinite set (sequence) of orthogonal projectors. Further, let $\hat{P}$ denote the limit of $\sum_{n=1}^{N} \hat{P}_{n}$, the corresponding ascending sequence $(N=1,2, \ldots, \infty)$ of sums of the orthogonal projectors (cf Remark 4 and (8)). Then $\hat{P}=\operatorname{lub}\left\{\hat{P}_{n}: n=1,2, \ldots, \infty\right\}$.

Proof. Let $|\psi\rangle$ be an arbitrary vector. By definition of convergence of a sequence of operators, one writes (in our case)

$$
\hat{P}|\psi\rangle=\lim _{N \rightarrow \infty} \sum_{n=1}^{N} \hat{P}_{n}|\psi\rangle .
$$


We have in mind that projectors are continuous (cf Lemma 2), i.e., they commute with every limit.

We first establish that $\hat{P}$ is an upper bound of the set $\left\{\hat{P}_{n}: n=1,2, \ldots, \infty\right\}$, i.e., that $\quad\left\{\hat{P}_{n} \leq \hat{P}: n=1,2, \ldots, \infty\right\} \quad$ (actually that $\left.\left\{\hat{P}_{n} \hat{P}=\hat{P}_{n}: n=1,2, \ldots, \infty\right\}\right)$ is valid. Let $\bar{n}$ be an arbitrary fixed index value

$\hat{P}_{\bar{n}} \hat{P}|\psi\rangle=\lim _{N \rightarrow \infty} \sum_{n=1}^{N} \hat{P}_{\bar{n}} \hat{P}_{n}|\psi\rangle=\lim _{N \rightarrow \infty} \sum_{n=1}^{N} \delta_{n, \bar{n}} \hat{P}_{n}|\psi\rangle=\hat{P}_{\bar{n}}|\psi\rangle$.

Finally we have to prove that $\hat{P}$ is the minimal upper bound. Let $\hat{Q}$ be an arbitrary upper bound of the set $\left\{\hat{P}_{n}: n=1,2, \ldots, \infty\right\}$ :

$$
\forall n: \quad \hat{Q} \hat{P}_{n}=\hat{P}_{n} .
$$

It is claimed that $\hat{Q} \hat{P}=\hat{P}$. To prove it, we write

$$
\hat{Q} \hat{P}|\psi\rangle=\lim _{N \rightarrow \infty} \sum_{n=1}^{N} \hat{Q} \hat{P}_{n}=\lim _{N \rightarrow \infty} \sum_{n=1}^{N} \hat{P}_{n}=\hat{P}|\psi\rangle .
$$

The last step is a consequence of 97 .

Definition 5. Let $\left\{\hat{P}_{n}: n=1,2, \ldots, N ; 2 \leq N \leq \infty\right\}$ be any (finite or countably infinite) set of orthogonal projectors: $\hat{P}_{n} \hat{P}_{n^{\prime}}=\hat{0}$ if $n \neq n^{\prime}$. Owing to the completelattice isomorphism (cf Theorem 3), which preserves orthogonality (cf Proposition 6) we have by implication: $\left\{\mathcal{R}\left(\hat{P}_{n}\right): n=1,2, \ldots, N\right\}, \mathcal{R}\left(\hat{P}_{n}\right) \perp \mathcal{R}\left(\hat{P}_{n^{\prime}}\right)$ if $n \neq n^{\prime}$ for the corresponding (in the sense of the basic bijection, cf Theorem 11) set of subspaces. Then the so-called $o r$ thogonal sums $\sum_{n=1}^{N} \hat{P}_{n}$ and $\sum_{n=1}^{\oplus, N} \mathcal{R}\left(\hat{P}_{n}\right)$ are defined as the lub of the given set of projectors and that of ranges respectively.

Proposition 7. Finite and countably infinite orthogonal sums are preserved in both directions by the basic bijection (cf Theorems 1 and 2).

Proof. In the preceding definition finite and countably infinite orthogonal sums are defined as least upper bounds. Since the basic bijection establishes an isomorphism of complete lattices (cf Theorem 3), least upper bounds are preserved in both directions.

Definition 6. Every projector $\hat{P}$ has an orthocomplementary projector (shortly: ortho-complement) that is orthogonal to $\hat{P}$ and its orthogonal sum with $\hat{P}$ is the identity operator. It is denoted by ' $\hat{P}^{\perp}$, , and $\hat{P}^{\perp} \equiv \hat{I}-\hat{P}$. Correspondingly, every subspace $\mathcal{R}(\hat{P})$ (cf Theorem 1 ) has an ortho-complement $\mathcal{R}\left(\hat{P}^{\perp}\right)$ such that $\mathcal{R}(\hat{P}) \perp \mathcal{R}\left(\hat{P}^{\perp}\right)$ and $\mathcal{R}(\hat{P}) \oplus \mathcal{R}\left(\hat{P}^{\perp}\right)=\mathcal{H}$. The ortho-complement $\mathcal{R}\left(\hat{P}^{\perp}\right)$ is also called the null space of $\hat{P}$ and denoted by $\mathcal{N}(\hat{P})$. The null space of $\hat{P}$ is the range of the ortho-complementary projector $\hat{P}^{\perp}$ and vice versa $[8]$.
Remark 5. Since orthogonality and orthogonal sums are preserved in the basic bijection in both directions (cf Propositions 6 and 7), so is taking the orthocomplement.

\subsection{Spanning}

Let us go back to $\mathcal{S}_{S}$, the complete lattice of all subspaces in a given Hilbert space (cf Proposition 5). Let $\mathcal{A}$ be an arbitrary set of subspaces. The very taking the lub of $\mathcal{A}$ one often expresses by saying that $\mathcal{A}$ spans the lub.

This term is used even more often if one has an arbitrary set of vectors $\{|n\rangle: n \in \mathcal{M}\}$. One says that the set spans a subspace having in mind the minimal subspace that contains the set.

\subsection{Certainty and impossibility}

As it is well known, in the formalism of quantum mechanics there are two kinds of basic entities. The first kind are the elements, usually called vectors, of the given Hilbert space. One mostly uses vectors of norm 1 and calls them state vectors. The second kind are the projectors, which are usually physically interpreted as events. If $|\psi\rangle$ is a state vector and $\hat{P}$ a projector, then the expression $\langle\psi|\hat{P}| \psi\rangle$ has the physical meaning of the probability that $\hat{P}$ will occur in a measurement of $\hat{P}$ in the state $|\psi\rangle$.

Lemma 8. (Certainty) Let $|\psi\rangle$ be a quantum state vector and $\hat{P}$ a projector (event). Certainty of the latter in the former can be expressed in three equivalent ways.

(i) The physical way: $\langle\psi|\hat{P}| \psi\rangle=1$;

(ii) The algebraic way: $\hat{P}|\psi\rangle=|\psi\rangle$;

(iii) The geometric way: $|\psi\rangle \in \mathcal{R}(\hat{P})$.

Proof. Follows the scheme (ii) $\Leftrightarrow$ (i) and (ii) $\Leftrightarrow$ (iii), which due to the fact that equivalence is transitive implies $(i) \Leftrightarrow($ iii). In the former part of the proof use will be made of the fact that only the zero vector has zero norm (cf (1)). (ii) $\Leftrightarrow(i)$ : This claim has been proved in Lemma 1 in view of the fact that state vectors have norm equal to 1 by definition. (ii) $\Leftrightarrow$ (iii): It has been proved in Proposition 2 that the range of any projector is its 1-eigen-subspace. This immediately implies the present claim.

Lemma 9. (Impossibility) Quantum-mechanical impossibility of an event (projector) $\hat{P}$ in a state vector $|\psi\rangle$ can be written in three equivalent ways.

(i) The physical way: $\langle\psi|\hat{P}| \psi\rangle=0$;

(ii) The algebraic way: $\hat{P}|\psi\rangle=0$;

(iii) The geometric way: $|\psi\rangle \in \mathcal{N}(\hat{P})$ where $\mathcal{N}(\hat{P}) \equiv \mathcal{R}(\hat{P})^{\perp}$ is the so-called null space (orthocomplement) of the range of $\hat{P}$. 
Proof. Impossibility of an event is equivalent to the certainty of the ortho-complementary event $\hat{P}^{\perp}=\hat{I}-\hat{P}$, for which the three relations in Lemma 8 are valid. The rest is then obvious.

\subsection{The lattice of two projectors and more}

Let $\hat{P}$ and $\hat{Q}$ be projectors. Then $(\hat{P} \hat{Q})^{\dagger}=\hat{Q} \hat{P}$. As it is seen, the product is self-adjoint if and only if the projectors commute. If they do, then

$$
(\hat{P} \hat{Q})^{2}=\hat{P} \hat{Q} \hat{P} \hat{Q}=\hat{P} \hat{Q} .
$$

Idempotency is implied. It is straightforward to upgrade the obvious conclusion by mathematical induction (cf proof of Lemma 5). Hence the following statement can be made.

Remark 6. Any finite number of projectors multiply into (their string of acting one after another is) a projector if and only if they all commute.

Let again $\hat{P}$ and $\hat{Q}$ be projectors. Their sum is selfadjoint. As to their square,

$$
(\hat{P}+\hat{Q})(\hat{P}+\hat{Q})=\hat{P}+\hat{P} \hat{Q}+\hat{Q} \hat{P}+\hat{Q} .
$$

As it is seen, commutation in itself does not imply idempotency. But orthogonality does (it implies also commutation). It is again straightforward to upgrade the conclusion by mathematical induction. Therefore the following claim is valid.

Remark 7. Any finite number of projectors add up to a projector if and only if they are two-by-two orthogonal. The orthogonal sum of any finite number of projectors is preserved by the basic bijection (cf Lemma 7 and Theorem 3).

Proposition 8. If two projectors $\hat{P}$ and $\hat{Q}$ commute, then their product $\hat{P} \hat{Q}$ is their common glb: glb $\{\hat{P}, \hat{Q}\}=\hat{P} \hat{Q}$; the projectors $(\hat{P}-\hat{P} \hat{Q})$ and $(\hat{Q}-\hat{P} \hat{Q})$ are orthogonal, and

$$
\operatorname{lub}\{\hat{P}, \hat{Q}\}=\hat{P} \hat{Q}+(\hat{P}-\hat{P} \hat{Q})+(\hat{Q}-\hat{P} \hat{Q}) .
$$

Proof. The assumed commutation (entailing idempotency of $\hat{P} \hat{Q}$ ) implies that $\hat{P} \hat{Q}$ absorbs both $\hat{P}$ and $\hat{Q}$ : $(\hat{P} \hat{Q}) \hat{P}=(\hat{P} \hat{Q}) \hat{Q}=\hat{P} \hat{Q}$. Hence, $\hat{P} \hat{Q}$ is a common lower bound of $\hat{P}$ and $\hat{Q}$. If so is another projector $\hat{R}$, then it absorbs also $\hat{P} \hat{Q}:(\hat{P} \hat{Q}) \hat{R}=\hat{R}$. Thus, $\hat{P} \hat{Q}$ is the greatest common lower bound: $g l b\{\hat{P}, \hat{Q}\}=\hat{P} \hat{Q}$. Next,

$$
(\hat{P}-\hat{P} \hat{Q})(\hat{Q}-\hat{P} \hat{Q})=\hat{P} \hat{Q}-\hat{P} \hat{Q}-\hat{P} \hat{Q}+\hat{P} \hat{Q}=\hat{0} .
$$

Finally, both $\hat{P}$ and $\hat{Q}$ absorb $\hat{P} \hat{Q}+(\hat{P}-\hat{P} \hat{Q})+(\hat{Q}-$ $\hat{P} \hat{Q}$ ) making the latter a common upper bound of $\hat{P}$ and
$\hat{Q}$. Further, let $\hat{R}$ be another common upper bound, i.e., let both $\hat{P}$ and $\hat{Q}$ absorb $\hat{R}$. Then (obviously) so does $\hat{P} \hat{Q}+(\hat{P}-\hat{P} \hat{Q})+(\hat{Q}-\hat{P} \hat{Q})$, which makes this projector the $\operatorname{lub}\{\hat{P}, \hat{Q}\}$ as claimed.

Lemma 10. If $\hat{P}$ and $\hat{Q}$ are orthogonal projectors, then their common glb is the zero projector $\hat{0}$.

Proof. Orthogonality implies commutation. Hence $\hat{0}=\hat{P} \hat{Q}=\hat{Q} \hat{P}=\operatorname{glb}(\hat{P}, \hat{Q})$.

Remark 8. In general, the product of two projectors is not preserved by the basic bijection (cf Theorem[1). But if the projectors commute, their product is the common $\mathrm{glb}$ (cf the preceding Proposition 8 , and as such it is preserved:

$$
\hat{P} \hat{Q}=\hat{Q} \hat{P} \quad \Rightarrow \quad \mathcal{R}(\hat{P} \hat{Q})=\mathcal{R}(\hat{P}) \cap \mathcal{R}(\hat{Q}) .
$$

Remark 9. If $\hat{P}$ and $\hat{Q}$ are two projectors, in general subtraction $\hat{P}-\hat{Q}$ is not preserved in the basic bijection. Nevertheless, if the latter is a sub-projector of the former, then it is preserved ( $\mathrm{cf}$ Theorem 2).

Lemma 11. The counterpart (corresponding statement) of (11) in terms of corresponding subspaces has the following form. If $\hat{P}$ and $\hat{Q}$ are two commuting projectors, then

$$
\begin{aligned}
\operatorname{lub}\{\mathcal{R}(\hat{P}), \mathcal{R}(\hat{Q})\}= & \mathcal{R}(\hat{P}) \cap \mathcal{R}(\hat{Q}) \\
& \oplus[\mathcal{R}(\hat{P})-\mathcal{R}(\hat{P}) \cap \mathcal{R}(\hat{Q})] \\
& \oplus[\mathcal{R}(\hat{Q})-\mathcal{R}(\hat{P}) \cap \mathcal{R}(\hat{Q})] .
\end{aligned}
$$

Proof. Since orthogonal sums are preserved in the basic bijection (cf Lemma 7 and Theorem 3), the counterpart of (11) is given by (13).

Lemma 12. If $\mathcal{B}$ is an arbitrary set of projectors containing two orthogonal projectors $\hat{P}, \hat{Q}, \hat{P} \hat{Q}=\hat{0}$, then $g l b(\mathcal{B})=\hat{0}$. If they additionally add up to the identity operator, then also lub $(\mathcal{B})=\hat{I}$.

Proof. Orthogonality of $\hat{P}$ and $\hat{Q}$ implies $g l b\{\hat{P}, \hat{Q}\}=\hat{0}$ (cf Lemma 10). Hence, $\hat{0}$ is the only lower bound of $\{\hat{P}, \hat{Q}\}$. Each lower bound of $\mathcal{B}$ is also a lower bound of $\{\hat{P}, \hat{Q}\}$, because $\{\hat{P}, \hat{Q}\} \subseteq \mathcal{B}$. Therefore $\mathcal{B}$ has $\hat{0}$ as its only lower bound, which is then its glb. Since orthogonal sum of projectors is their lub (cf Definition 5), the analogous (symmetric) argument proves the second claim.

\subsection{Canonical spectral decomposition of discrete observables}

Perhaps the most important application of this review is the canonical spectral decomposition of any discrete selfadjoint operator (operator with a purely discrete spectrum, i.e., with no continuous spectrum). It may represent an observable or be any density operator. 
Definition 7. Let $\hat{O}$ be a discrete self-adjoint operator, $\left\{o_{n}: n=1,2, \ldots, N\right\}$ its spectrum (set of all eigenvalues), $N$ finite or $N=\infty$ ( $N$ countably infinite), and $\left\{\hat{E}_{n}: n=1,2, \ldots, N\right\}$ the set of its corresponding eigenprojectors.

The basic orthogonal sum

$$
\sum_{n=1}^{N} \hat{E}_{n}=\hat{I},
$$

is called the completeness relation or the spectral decomposition of the identity operator. Then

$$
\hat{O}=\sum_{n=1}^{N} o_{n} \hat{E}_{n}
$$

is called the canonical spectral decomposition of $\hat{O}$ (as opposed to, e.g., spectral decomposition into spectral ray projectors, i.e., projectors of one-dimensional ranges, or any other intermediary case of decomposing the eigenprojectors). One should note that only the canonical decomposition is in terms of eigenvalues that are all distinct. All the other spectral decompositions contain repetition of same eigenvalues due to decomposition of the corresponding eigen-projectors ( $\mathrm{cf}[14]$ ).

Returning to the basic orthogonal sum (14), its isomorphic image ( $\mathrm{cf}$ Theorem 1 and Theorem 3 ) is the orthogonal decomposition of the Hilbert space into the corresponding eigen-subspaces:

$$
\sum_{n=1}^{\oplus, N} \mathcal{R}\left(\hat{E}_{n}\right)=\mathcal{H} .
$$

Remark 10. A well-known example is the operator representing the Hamiltonian of a quantum system. In the excitation spectrum one sometimes finds parts of too dense or too numerous (perhaps even infinitely many) so-called bands of energy levels. The instruments in the laboratory may not be able to handle the individual levels in such bands. The bands are then treated as single levels. Theoretically this corresponds to replacing the orthogonal sums of some successive eigen-projectors in the bands by single projectors.

Remark 11. One should note that in case of an infinite spectrum of the above discrete self-adjoint operator $\hat{O}$, we have 3 infinite sums (14)-(16). Following von Neumann [5], the standard quantum-mechanical formalism defines all three as limits of sequences of finite sums. The approach of this review, laying emphasis on the isomorphism of complete lattices (Theorem 3), has established an equivalent alternative definition of the infinite sums (14) and (16) as least upper bounds of the set of all terms. But (15) has no alternative.
Remark 12. Let us consider the case of a self-adjoint operator $\hat{O}$ that has both a discrete part of its spectrum and a continuous part. (The purely continuous case is completely outside the scope of this review.) It is known in general spectral theory in Hilbert space that $\sum_{n=1}^{\oplus, N} \mathcal{R}\left(\hat{E}_{n}\right)$, the orthogonal sum of the ranges of all eigen-projectors is an invariant subspace for $\hat{O}$. The operator reduces in it (is acting in it) as a discrete operator.

\section{Conclusion}

Considering the Hilbert-space projectors, which play a central role in the quantum-mechanical formalism [6, 15, 16], without their ranges (purely algebraic treatment) makes their lattice properties hidden. Including the ranges, however, exhibits these lattice properties in an explicit way.

A summary of the main issues: the subspace property of the projector ranges (as opposed to the mere linearmanifold property of the other operators), and its invertibility have made the basic bijection in Theorem 1 the first important and basic issue. The partial-order relations among projectors (the sub-projector relation) and that among subspaces (the subset relation) have made possible upgrading the basic bijection into an isomorphism of partially-ordered sets (Theorem 2). The completelattice property of the set of all subspaces (demonstrated in Proposition 5 leaning on Proposition 44 has then implied the complete-lattice property of the set of all projectors (Theorem 3). Application to the infinite sums of eigen-projectors and eigen-subspaces of discrete observables is one of the main issues of this review. Ample use is made of the powerful mirror-image-like property of isomorphism: whatever is valid in terms of the isomorphic mathematical structure in one of the sets is necessarily valid also in the isomorphic set in terms of the isomorphic entities. Equivalence of the derived complete-lattice definition of the mentioned infinite sums with the standard formalism in terms of converging ascending projector sequences is presented and proved in Theorem 4, It has established balance between the reviewed aspect of the quantum-mechanical formalism (projectors versus subspaces) and the mostly used parts of the formalism.

The final section on the canonical spectral decomposition of any discrete self-adjoint operator deals with actual operative quantum mechanics, rather then with pure formalism. Among other aims, it highlights the fact that the quantum-mechanical formalism is not just mathematics. It is an indispensable part of quantum mechanics. It is its language, its set of rules, or even set of laws.

The reader who made use of the quantum formalism may object that he did not encounter a partial-order rela- 
tion so often or none at all. But the series of arguments in the present review has hopefully made it clear that a full answer to the relation between projectors and subspaces puts the partial-order relation in a central position (though practical use of the formalism may mostly circumvent this fact).

In quantum logic the quantum-mechanical projectors have the third physical meaning (besides event and property): quantum statement. The eigenvalue 1 means that the given statement is true. The eigenvalue 0 means that the given statement is not true. And there are the indeterminate cases. In quantum logic the sub-projector relation is called 'quantum logical implication'. The series of arguments in this review has shown that the set of all quantum statements is a complete lattice regarding quantum logical implication.

\section{Acknowledgment}

I am grateful to both editors of this article for their helpful suggestions for improvement and, aware of my eye-sight problems, generously sacrificed their personal time and efforts to make my manuscript publishable.

\section{References}

[1] G. Birkhoff, J. Von Neumann. The logic of quantum mechanics. Annals of Mathematics 1936; 37(4):823843. doi : 10.2307/1968621.

[2] G. Birkhoff. Lattice Theory. 3rd Edition. Vol. 25 of Colloquium Publications. American Mathematical Society, New York, 1973.

[3] K. Bugajska, S. Bugajski. The lattice structure of quantum logics. Annales de l'Institut Henri Poincaré. Section A, Physique théorique 1973; 19(4):333-340. http : //wWW . numdam . org/item/ AIHPA_1973__19_4_333_0, MR:359556.

[4] A. Wilce. Quantum logic and probability theory. in: E. N. Zalta (Ed.), Stanford Encyclopedia of Philosophy. Center for the Study of Language and Information (CSLI), Stanford University, Stanford, California, 2017. https://plato.stanford.edu/ entries/qt-quantlog/.

[5] J. von Neumann. Mathematical Foundations of Quantum Mechanics. Princeton University Press, Princeton, 1955.
[6] P. A. M. Dirac. The Principles of Quantum Mechanics. 4th Edition. Oxford University Press, Oxford, 1967. https://archive.org/details/ DiracPrinciples0fQuantumMechanics.

[7] P. A. M. Dirac. A new notation for quantum mechanics. Mathematical Proceedings of the Cambridge Philosophical Society 1939; 35(3):416-418. doi : 10.1017/SO305004100021162

[8] W. G. Strang. Introduction to Linear Algebra. 5th Edition. Wellesley-Cambridge Press, Wellesley, Massachusetts, 2016.

[9] C. A. Hooker. The Logico-Algebraic Approach to Quantum Mechanics: Volume I: Historical Evolution. Vol. 5a of The Western Ontario Series in Philosophy of Science. D. Reidel, Dordrecht, 1975. doi : 10.1007/978-94-010-1795-4.

[10] S. Roman. Lattices and Ordered Sets. Springer, New York, 2008. doi:10.1007/ 978-0-387-78901-9.

[11] J. Ganapathy. Maximal elements and upper bounds in posets. Pi Mu Epsilon Journal 1992; 9(7):462464. JSTOR: 24340068 .

[12] G. Grätzer. Lattice Theory: Foundation. Birkhäuser, Basel, 2011. doi:10.1007/ 978-3-0348-0018-1.

[13] V. S. Varadarajan. Geometry of Quantum Theory. 2nd Edition. Springer, New York, 2007. doi : 10. 1007/978-0-387-49386-2.

[14] L. Marchildon. Quantum Mechanics: From Basic Principles to Numerical Methods and Applications. Advanced Texts in Physics. Springer, Berlin, 2002. doi : 10.1007/978-3-662-04750-7.

[15] J. Blank, P. Exner, M. Havlíček. Hilbert Space Operators in Quantum Physics. Theoretical and Mathematical Physics. Springer, Berlin, 2008. doi: 10. $1007 / 978-1-4020-8870-4$.

[16] G. Fano, S. M. Blinder. Twenty-First Century Quantum Mechanics: Hilbert Space to Quantum Computers: Mathematical Methods and Conceptual Foundations. UNITEXT for Physics. Springer, Cham, Switzerland, 2017. doi : 10.1007/ 978-3-319-58732-5 